\title{
State-constrained optimal control of phase-field equations with obstacle
}

\author{
Jiashan Zheng ${ }^{1}$, Junjun Liu ${ }^{1}$ and Hao Liu²*
}

"Correspondence:

liuhao123@ustl.edu.cn

${ }^{2}$ School of Science, University of Science and Technology Liaoning, Anshan, 114051, China

Full list of author information is available at the end of the article

\begin{abstract}
This paper is concerned with an optimal control problem for the phase-field transition system with state constraint and obstacle. After showing the relationship between the control problem and its approximation, we derive Pontryagin's maximum principle for an optimal control of our original problem by using one of the approximate problems.
\end{abstract}

MSC: 49K20; 49J20; 74N25

Keywords: phase-field transition system; optimal control; Pontryagin's maximum principle

\section{Introduction}

We consider the optimal control of solid-liquid phase transitions:

$$
\left\{\begin{array}{l}
u_{t}-\gamma \Delta u+\delta \partial I_{[-1,1]}(u)+\varphi(u)-\lambda^{\prime}(u) v \ni 0 \quad \text { in } Q=\Omega \times(0, T) \\
v_{t}+(\lambda(u))_{t}-k \Delta v=B w \quad \text { in } Q=\Omega \times(0, T), \\
u(x, 0)=u_{0}(x), \quad v(x, 0)=v_{0}(x) \quad \text { in } \Omega \\
\frac{\partial u}{\partial v}=\frac{\partial v}{\partial v}=0 \quad \text { on } \Sigma=\partial \Omega \times(0, T)
\end{array}\right.
$$

and

$$
F(u) \subset S,
$$

where $\Omega$ is a bounded domain in $R^{N}(1 \leq N \leq 3)$ with a smooth boundary $\partial \Omega, \varphi(u)=$ $u^{3}-u, \delta, \gamma, k>0$ denote given parameters, $\partial I_{[-1,1]}(u)$ is the subdifferential of the indicator function $I_{[-1,1]}(u)$ on the closed interval $[-1,1], B w$ is a given forcing term on $Q, \frac{\partial}{\partial v}$ is the outward normal derivative on $\partial \Omega$ and $u_{0}, v_{0}$ are given initial datums.

System (1.1) is a simplified model for a class of solid-liquid phase change problems. In the context of solid-liquid phase transitions, $v$ and $u$ represent the absolute temperature and the order parameter which indicates the physical situation of the system, respectively. Therefore it is natural to assume that the range of $u$ is bounded, say the closed interval $[-1,1]$ in this paper, and $\partial I_{[-1,1]}(u)$ denoting the range of the order parameter $u$ is assumed to be a compact interval $[-1,1], u(t, x) \equiv-1$ and $u(t, x) \equiv 1$ mean, respectively, that the physical situation at $(t, x)$ is of pure solid and pure liquid, while $-1<u(t, x)<1$ means that the physical situation at $(t, x)$ is mushy.

@2013 Zheng et al.; licensee Springer. This is an Open Access article distributed under the terms of the Creative Commons Attribution License (http://creativecommons.org/licenses/by/2.0), which permits unrestricted use, distribution, and reproduction in any medium, provided the original work is properly cited. 
A great deal of research has been done on the phase-field transition system, for which we refer to the book by Temam [1] and the references therein. Without the term $\partial I_{[-1,1]}(u)$, system (1.1) is the standard phase field model which was studied in $[2,3]$. One of the most important characteristics of our model is the nonlinear term $\partial I_{[-1,1]}(u)$ (obstacle) which allows the coexistence of pure phases in the dynamical phase transition process. The existence and uniqueness of solution for the phase field model with obstacle were discussed in [4-8]. In particular, the asymptotic behavior of solutions to the non-isothermal phasefield transition system with obstacle was considered in [6] and [7]. Recently, the Caginalp phase-field system with coupled dynamic boundary conditions, including the singular potentials, was presented in [9] and [10].

Throughout this paper, the Hilbert space $H=L^{2}(\Omega)$ is equipped with the usual inner product $(\cdot, \cdot)$ and the norm $|\cdot|_{2}$. Define a closed subspace $H_{0}$ of $H$ by $H_{0}=\left\{z \in H ; \int_{\Omega} z d x=\right.$ $0\}$. We put $V_{0}=V \cap H_{0}$ with $\|v\|_{V_{0}}=|\nabla v|_{L^{2}(\Omega)} \equiv\|v\|_{2}$, where $V=\left\{v \in H^{1}(\Omega), \frac{\partial v}{\partial v}=0\right\}$. If we identify $H^{*}$ and $H_{0}^{*}$ with their dual space, then we have $V \subset H \subset V^{*}$ and $V_{0} \subset H_{0} \subset V_{0}^{*}$, where $V_{0}^{*}$ is the duality space of $V_{0}$.

Throughout the paper, we suppose that the following assumptions hold.

Let $U$ be a real Hilbert space, $B: U \rightarrow H$ be a linear continuous operator. Assuming that $Z$ is a Banach space with the dual $Z^{*}$ strictly convex, let $S \subset Z$ be a closed convex subset with finite co-dimensionality.

$\left(\mathrm{H}_{1}\right) \quad F: L^{2}(0, T ; H) \rightarrow Z$ is in the class of $C^{1}$.

$\left(\mathrm{H}_{2}\right) g:[0, T] \times H \rightarrow R^{+}$is measurable in $t$ and for every $\sigma>0$, there exists $L_{\sigma}>0$ independent of $t$ such that $g(0, u) \in L^{\infty}(0, T)$ and

$$
|g(t, y)-g(t, z)| \leq L_{\sigma}|y-z|_{2} \quad \text { for any } t \in[0, T] \text { with }|y|_{2}+|z|_{2} \leq \lambda \text {. }
$$

$\left(\mathrm{H}_{3}\right) h: U \rightarrow \bar{R}$ is lower semicontinuous and convex with the following growth property:

$$
h(w) \geq c_{1}|w|_{U}^{2}+c_{2} \quad \text { for any } w \in U \text { with } c_{1}>0 \text { and } c_{2} \in R \text {. }
$$

$\left(\mathrm{H}_{4}\right) \lambda \in C^{2}$ and there exists a constant $\kappa>0$ such that $\lambda^{\prime \prime}(s) \leq \kappa$ for any $s \in R$.

We consider the following optimal control problem:

$$
\min L(w) \quad \text { over all } w \in L^{2}(0, T ; U)
$$

where

$$
L(w)=\int_{0}^{t}[g(t, u(t))+h(w(t))] d t
$$

and

$$
(u, v) \text { is the solution of (1.1) corresponding to } w, F(u) \subset S \text {. }
$$

For any

$$
(u, v, w) \in Y \times Y \times L^{2}(0, T ; U)
$$

satisfying (1.1) is called a feasible pair, where $Y=H^{2,1}(Q) \cap C(0, T ; V)$. 
The first question regarding problem $(P)$ is if there is an admissible solution, i.e., if the set $A_{a d}$ is nonempty. Taking into account [11] similarly, we may assume in the sequel that for $(u, v, w) \in Y \times Y \times L^{2}(0, T ; U)$, problem $(P)$ admits at least one admissible solution.

Optimal control problems of the phase transition system have been studied by several authors (for instance, see [12-16]). In particular, let $\lambda(u)=\frac{l}{2}$ and $\delta=0$ in (1.1), the optimal boundary controls for a phase field model and the state-constrained optimal control for the phase-field transition system were considered in [13] and [15], respectively. In [16], based on the energy estimates and the compact method, Ryu and Yagi considered the optimal control problems of the adsorbate-induced phase transition model. It is noted that the optimal control without state constraint or without obstacle of the phase field model was discussed in [14, 17-20].

To the best of our knowledge, there are few papers concerned with the optimal control problems for the phase-field with obstacle although it is natural to have the obstacle in the solid-liquid phase transitions and related physics models, since the obstacle $\partial I_{[-1,1]}(u)$ brings the essential difficulty in getting Pontryagin's maximum principle for corresponding models.

We state the maximum principle as follows.

Theorem 1.1 Suppose that $\left(\mathrm{H}_{1}\right),\left(\mathrm{H}_{2}\right),\left(\mathrm{H}_{3}\right)$ and $\left(\mathrm{H}_{4}\right)$ hold. Let $\left(u^{*}, v^{*}, w^{*}\right)$ be optimal for problem $(P)$, then there exists a tetrad $\left(\mu_{0}, p, q, \zeta_{0}\right) \in R \times L^{2}(0, T ; V) \cap L^{\infty}(0, T ; H) \times$ $L^{2}(0, T ; V) \cap L^{\infty}(0, T ; H) \times Z^{*}$ with $\left(\mu_{0}, \zeta_{0}\right) \neq 0$ and a measure $\eta \in L^{\infty}(Q)^{*}$ such that

$$
\left\{\begin{array}{l}
-p_{t}-\gamma \Delta p+\eta+\left(3\left(u^{*}\right)^{2}-1\right) p-\lambda^{\prime \prime}\left(u^{*}\right) p v^{*}-\lambda^{\prime}\left(u^{*}\right) q \\
\quad \in-\left[\partial F\left(u^{*}\right)\right]^{*} \zeta_{0}-\mu_{0} \partial g\left(t, u^{*}\right), \\
-q_{t}+\lambda^{\prime}\left(u^{*}\right) p_{t}-k \Delta q+\lambda^{\prime \prime}\left(u^{*}\right) p u_{t}^{*}=0 \\
p(T)=0, \quad q(T)=0
\end{array}\right.
$$

and

$$
\left\{\begin{array}{l}
B^{*} q(t) \in \mu_{0} \partial h\left(w^{*}(t)\right), \\
\left\langle\zeta_{0}, s-\partial F\left(u^{*}\right)\right\rangle_{Z^{*}, Z} \leq 0 \quad \forall s \in S .
\end{array}\right.
$$

Moreover, if $F^{\prime}\left(u^{*}\right)$ is injective, then $\left(\mu_{0}, p, q\right) \neq 0$.

The rest of this paper is organized as follows. In Section 2, we provide existence results and $a$ priori estimates in the form that is required to obtain Pontryagin's maximum principle for problem $(P)$. Besides the existence of an optimal control in problem $\left(P^{\varepsilon}\right)$, necessary optimality conditions for this problem and for problem $(P)$ are proved in Section 3.

\section{The approximation problem}

This section is to show the existence of the optimal control of the approximation problem corresponding to the phase transition system. To this end, we first show some technical lemmas, which are presented below for the sake of completeness.

In order to approximate $\partial I_{[-1,1]}(\cdot)$, we define a nondecreasing function $\beta^{\varepsilon}[21]$ on $R$ by

$$
\beta^{\varepsilon}(r)=\operatorname{sign}(r) \int_{0}^{|r|} \min \left\{\frac{1}{\varepsilon}, \frac{[s-1]^{+}}{\varepsilon^{2}}\right\} d s \quad \forall r \in R,
$$


where $[\cdot]^{+}$denotes the positive part of functions. Then $\beta^{\varepsilon} \in C^{1},\left(\beta^{\varepsilon}\right)^{\prime} \in W^{1, \infty}(R)$ and

$$
0 \leq\left(\beta^{\varepsilon}\right)^{\prime}(r) \leq \frac{1}{\varepsilon}, \quad\left|\left(\beta^{\varepsilon}\right)(r)\right| \geq \frac{1}{\varepsilon}\left([r-1]^{+}+[-1-r]^{+}\right)-\frac{1}{2} \quad \text { for any } r \in R .
$$

We fix a primitive $\hat{\beta}^{\varepsilon}$ of $\beta^{\varepsilon}$ such that

$$
\hat{\beta}^{\varepsilon}(0)=0 \quad \text { and } \quad \hat{\beta}^{\varepsilon}(r) \geq 0 \quad \text { for any } r \in R .
$$

Without loss of generality, we may assume $\delta=1$, therefore, the approximation of (1.1) is

$$
\left\{\begin{array}{l}
u_{t}-\gamma \Delta u+\beta^{\varepsilon}(u)+\varphi(u)-\lambda^{\prime}(u) v=0 \quad \text { in } Q=\Omega \times(0, T), \\
v_{t}+\lambda^{\prime}(u) u_{t}-k \Delta v=B w \quad \text { in } Q=\Omega \times(0, T), \\
u(x, 0)=u_{0}(x), \quad v(x, 0)=v_{0}(x) \quad \text { in } \Omega, \\
\frac{\partial u}{\partial v}=\frac{\partial v}{\partial v}=0 \quad \text { on } \Sigma=\partial \Omega \times(0, T) .
\end{array}\right.
$$

Lemma 2.1 Suppose that $\beta^{\varepsilon}(\cdot)$ satisfies (2.1)-(2.2), $w_{n} \in L^{2}(0, T ; U), w_{n} \rightarrow \tilde{w}$ weakly in $L^{2}(0, T ; U)$ and $(\tilde{u}, \tilde{v}),\left(u_{n}, v_{n}\right)$ are the solutions of $(2.3)$ corresponding to $\tilde{w}$ and $w_{n}$, respectively. Then there exists a subsequence of $\left(u_{n}, v_{n}\right)$, still denoted by itself, such that

$$
\begin{aligned}
& \left(u_{n}, v_{n}\right) \rightarrow(\tilde{u}, \tilde{v}) \quad \text { weakly in } Y^{2}, \\
& \beta^{\varepsilon}\left(u_{n}\right) \rightarrow \tilde{\eta} \quad \text { weakly in } L^{2}(0, T ; H) \text { as } n \rightarrow \infty .
\end{aligned}
$$

Proof Replacing $(u, v)$ and $w$ by $\left(u_{n}, v_{n}\right)$ and $w_{n}$ in (2.3), respectively, we obtain

$$
\left\{\begin{array}{l}
u_{n, t}-\gamma \Delta u_{n}+\beta^{\varepsilon}\left(u_{n}\right)+\varphi\left(u_{n}\right)-\lambda^{\prime}\left(u_{n}\right) v_{n}=0 \quad \text { in } Q=\Omega \times(0, T), \\
v_{n, t}+\lambda^{\prime}\left(u_{n}\right) u_{n, t}-k \Delta v_{n}=B w_{n} \quad \text { in } Q=\Omega \times(0, T), \\
u_{n}(x, 0)=u_{0}(x), \quad v_{n}(x, 0)=v_{0}(x) \quad \text { in } \Omega \\
\frac{\partial u_{n}}{\partial v}=\frac{\partial v_{n}}{\partial v}=0 \quad \text { on } \Sigma=\partial \Omega \times(0, T) .
\end{array}\right.
$$

Multiplying (2.5) ${ }_{1}$ and (2.5) $)_{2}$ by $u_{n, t}$ and $v_{n}$, respectively, integrating over $\Omega$ and adding the resulting equations, we end up with

$$
\begin{aligned}
& \frac{d}{d t}\left(\frac{\gamma}{2}\left|\nabla u_{n}\right|_{2}^{2}+\left|v_{n}\right|_{2}^{2}\right)+\left|u_{n, t}\right|_{2}^{2}+k\left|\nabla v_{n}\right|_{2}^{2}+\int_{\Omega}\left(\beta^{\varepsilon}\left(u_{n}\right)+\varphi\left(u_{n}\right)\right) u_{n, t} d x \\
& \quad \leq-\int_{\Omega} B w_{n} v_{n} d x .
\end{aligned}
$$

Therefore, we conclude with the help of Young's inequality and the properties of $\beta^{\varepsilon}$ that

$$
\begin{aligned}
& \frac{d}{d t}\left(\frac{\gamma}{2}\left|\nabla u_{n}\right|_{2}^{2}+\left|v_{n}\right|_{2}^{2}+\frac{1}{4}\left|u_{n}\right|_{L^{4}}^{4}+\left|\hat{\beta}^{\varepsilon}\right|_{L^{1}}\right)+k\left|\nabla v_{n}\right|_{2}^{2}+\left|u_{n, t}\right|_{2}^{2} \\
& \quad \leq \frac{1}{4}\left|B w_{n}\right|_{2}^{2}+\left|v_{n}\right|_{2}^{2}+\frac{1}{2}\left|u_{n, t}\right|_{2}^{2}+\frac{1}{2}\left|u_{n}\right|_{2}^{2} \\
& \quad \leq \frac{1}{4}\left|B w_{n}\right|_{2}^{2}+\left|v_{n}\right|_{2}^{2}+\frac{1}{2}\left|u_{n, t}\right|_{2}^{2}+\left|u_{n}\right|_{L^{4}}^{4}+C .
\end{aligned}
$$


Here and throughout the proof of Lemma 2.1, we shall denote by $C$ several positive constants independent of $n$. Applying Gronwall's inequality to (2.7), we derive

$$
\left|u_{n}\right|_{L^{\infty}\left(0, T ; V \cap L^{4}\right)}+\left|v_{n}\right|_{L^{\infty}(0, T ; H)}+\left|v_{n}\right|_{L^{2}(0, T ; V)}+\left|u_{n}\right|_{H^{1}(0, T ; H)}+\left|\hat{\beta}^{\varepsilon}\right|_{L^{\infty}\left(0, T ; L^{1}\right)} \leq C .
$$

Now, testing $(2.5)_{1}$ by $-\Delta u_{n}$, we derive

$$
\begin{aligned}
& \frac{1}{2} \frac{d}{d t}\left|\nabla u_{n}\right|_{2}^{2}+\gamma\left|\triangle u_{n}\right|_{2}^{2}+\int_{\Omega}\left(\beta^{\varepsilon}\right)^{\prime}\left|\nabla u_{n}\right|^{2}+3 u_{n}^{2}\left|\nabla u_{n}\right|^{2} d x \\
& \quad=-\int_{\Omega} \lambda^{\prime}\left(u_{n}\right) v_{n} \triangle u_{n} d x \\
& \quad \leq \frac{\gamma}{2}\left|\triangle u_{n}\right|_{2}^{2}+C\left(\left|v_{n}\right|_{L^{6}}^{6}+\int_{\Omega}\left|\lambda^{\prime}\left(u_{n}\right)\right|^{3} d x\right),
\end{aligned}
$$

which together with $\lambda^{\prime \prime}(t) \leq \kappa,(2.2),(2.8)$, Nirenberg's inequality and Gronwall's inequality implies that

$$
\left|u_{n}\right|_{L^{\infty}(0, T ; V)}+\left|u_{n}\right|_{L^{2}\left(0, T ; H^{2}\right)} \leq C
$$

Next, multiplying $(2.5)_{1}$ by $\beta^{\varepsilon}$, integrating over $[0, T]$ and invoking Young's inequality, we derive

$$
\begin{aligned}
& \frac{d}{d t}\left|\hat{\beta}^{\varepsilon}\right|_{L^{1}}+\left|\beta^{\varepsilon}\right|_{2}^{2} \\
& \quad=\int_{\Omega}\left(\gamma \Delta u_{n}-\varphi\left(u_{n}\right)+\lambda^{\prime}\left(u_{n}\right) v_{n}\right) \beta^{\varepsilon}\left(u_{n}\right) d x \\
& \quad \leq \frac{1}{2}\left|\beta^{\varepsilon}\right|_{2}^{2}+C\left(\left|u_{n}\right|_{L^{6}}^{6}+\left|u_{n}\right|_{2}^{2}+\gamma^{2}\left|\Delta u_{n}\right|_{2}^{2}+\int_{\Omega}\left|\lambda^{\prime}\left(u_{n}\right)\right|^{3} d x+\left|v_{n}\right|_{L^{6}}^{6}\right) .
\end{aligned}
$$

Thanks to (2.8), $\lambda^{\prime \prime}(t) \leq \kappa$ and Gronwall's inequality, we derive

$$
\left|\hat{\beta}^{\varepsilon}\right|_{L^{\infty}\left(0, T ; L^{1}\right)}+\left|\beta^{\varepsilon}\right|_{L^{2}(0, T ; H)} \leq C
$$

Inserting (2.10) and (2.12) into (2.5) 1 , we have

$$
\left|u_{n, t}\right|_{L^{2}(0, T ; H)} \leq C
$$

Now, differentiating $(2.5)_{1}$ with respect to $t$ and multiplying the result by $u_{n, t}$, then multiplying $(2.5)_{2}$ by $v_{t}$, adding the resulting equations and integrating over $\Omega$ leads to

$$
\begin{aligned}
& \frac{d}{d t}\left(\frac{1}{2}\left|u_{n, t}(t)\right|_{2}^{2}+\frac{k}{2}\left|\nabla v_{n}(t)\right|_{2}^{2}\right)+\gamma\left|\nabla u_{n, t}\right|_{2}^{2}+\int_{\Omega}\left(\beta^{\varepsilon}\right)^{\prime} u_{n, t}^{2}(x, t) d x+\left|v_{n, t}\right|_{2}^{2} \\
& \quad \leq\left|u_{n, t}(t)\right|_{2}^{2}+\int_{\Omega} \kappa v_{n}(t) u_{n, t}^{2}(t) d x+\int_{\Omega} B w_{n} v_{n, t} d x \\
& \quad \leq\left|u_{n, t}(t)\right|_{2}^{2}+\int_{\Omega}\left|\kappa v_{n}(t) u_{n, t}^{2}(t)\right| d x+\frac{1}{2}\left|v_{n, t}(t)\right|_{2}^{2}+\frac{1}{2}\left|B w_{n}\right|_{2}^{2} .
\end{aligned}
$$


On the other hand, with the help of (2.8), (2.10), Hölder's inequality and Nirenberg's inequality, we get

$$
\begin{aligned}
& \left|\int_{0}^{t} \int_{\Omega} \kappa v_{n}(t) u_{n, t}^{2} d x\right| \\
& \quad \leq|\kappa| \int_{0}^{t}\left|v_{n}(s)\right|_{L^{6}}\left|u_{n, t}(s)\right|_{L^{\frac{12}{5}}}^{2} d s \\
& \quad \leq|\kappa|\left(\int_{0}^{t}\left|v_{n}(s)\right|_{L^{6}}^{2} d s\right)^{\frac{1}{2}}\left(\int_{0}^{t}\left|u_{n, t}(s)\right|_{L^{\frac{12}{5}}}^{4} d s\right)^{\frac{1}{2}} \\
& \quad \leq C_{1}\left(\int_{0}^{t}\left|v_{n}(s)\right|_{H^{1}}^{2} d s\right)^{\frac{1}{2}} \times\left(\int_{0}^{t}\left(\left|\nabla u_{n, t}(s)\right|\left|u_{n, t}(s)\right|^{3}+\left|u_{n, t}(s)\right|^{4}\right) d s\right)^{\frac{1}{2}} \\
& \quad \leq C_{2} \sup _{0 \leq s \leq t}\left|u_{n, t}(s)\right| \times\left(\int_{0}^{t}\left(\left|\nabla u_{n, t}(s)\right|\left|u_{n, t}(s)\right|+\left|u_{n, t}(s)\right|^{2}\right) d s\right)^{\frac{1}{2}} \\
& \quad \leq C_{3} \sup _{0 \leq s \leq t}\left|u_{n, t}(s)\right|\left[1+\left(\int_{0}^{t}\left|\nabla u_{n, t}(s)\right|^{2} d s\right)^{\frac{1}{4}}\right] \\
& \quad \leq \mu \sup _{0 \leq s \leq t}\left|u_{n, t}(s)\right|_{2}^{2}+\mu \int_{0}^{t}\left|\nabla u_{n, t}(s)\right|^{2} d s+C_{4},
\end{aligned}
$$

where $\mu$ is a small positive constant and $C_{i}(i=1,2,3,4)$ are independent of $n$. Inserting (2.15) into (2.14), we derive

$$
\begin{aligned}
& \frac{1}{2}\left|u_{n, t}(t)\right|_{2}^{2}+\frac{k}{2}\left|\nabla v_{n}(t)\right|_{2}^{2} \\
& \quad+(\gamma-\mu)\left|\nabla u_{n, t}(t)\right|_{2}^{2}+\int_{0}^{t} \int_{\Omega}\left(\beta^{\varepsilon}\right)^{\prime}\left(u_{n}(t)\right) u_{n, t}^{2}(t) d x d s \\
& \leq \int_{0}^{t}\left|u_{n, s}(s)\right|_{2}^{2} d s+\mu \sup _{0 \leq s \leq t}\left|u_{n, t}(s)\right|_{2}^{2}+\frac{1}{2} \int_{0}^{t}\left|B w_{n}(s)\right|_{2}^{2} d s+C .
\end{aligned}
$$

Taking the supremum with respect to $t$ in (2.16), choosing $\mu>0$ sufficiently small and applying Gronwall's inequality, we end up with

$$
\sup _{0 \leq s \leq t}\left|u_{n, t}(t)\right|_{2}+\sup _{0 \leq s \leq t}\left|\nabla v_{n}(t)\right|_{2}+\left|\nabla u_{n, t}(t)\right|_{2}^{2} \leq C_{T}
$$

which combined with (2.13) implies that $\lambda^{\prime}\left(u_{n}\right) u_{n, t} \in L^{2}(0, T ; H)$. Therefore, employing the standard parabolic theory to $(2.5)_{2}$ leads to

$$
\left|v_{n, t}\right|_{L^{2}(0, T ; H)}+\left|v_{n}\right|_{L^{\infty}(0, T ; V)}+\left|v_{n}\right|_{L^{2}\left(0, T ; H^{2}\right)} \leq C
$$

Now we may combine the estimates (2.8), (2.10), (2.12), (2.13) and (2.18) to conclude the results. This completes the proof.

Lemma 2.2 Suppose that $\beta^{\varepsilon}(\cdot)$ satisfies (2.1)-(2.2), let $w_{\varepsilon} \in L^{2}(0, T ; U)$ with $w_{\varepsilon} \rightarrow w^{*}$ weakly in $L^{2}(0, T ; U)$ as $\varepsilon \rightarrow 0,\left(u_{\varepsilon}, v_{\varepsilon}\right)$ be the solution of (2.3) corresponding to $w_{\varepsilon}$. Then, on some subsequence $\left(u_{\varepsilon_{n}}, v_{\varepsilon_{n}}\right)$ of $\left(u_{\varepsilon}, v_{\varepsilon}\right)$, there exists a quad $(u, v, \eta) \in Y \times Y \times L^{2}(0, T ; H)$ 
such that

$$
\eta \in \partial I_{[-1,1]}(u) \text { a.e. } L^{2}(0, T ; H)
$$

while

$$
\begin{aligned}
& \left(u_{\varepsilon_{n}}, v_{\varepsilon_{n}}\right) \rightarrow(u, v) \quad \text { weakly in }\left(L^{\infty}(0, T ; V) \cap L^{2}\left(0, T ; H^{2}(\Omega)\right)\right)^{2}, \\
& \left(u_{\varepsilon_{n}}, v_{\varepsilon_{n}}\right) \rightarrow(u, v) \quad \text { strongly in }\left(L^{2}(0, T ; V) \cap C(0, T ; H)\right)^{2}, \\
& \left(u_{\varepsilon_{n}}^{\prime}, v_{\varepsilon_{n}}^{\prime}\right) \rightarrow\left(u^{\prime}, v^{\prime}\right) \quad \text { weakly in }\left(L^{2}(0, T ; H)\right)^{2}, \\
& \beta^{\varepsilon}\left(u_{\varepsilon_{n}}\right) \rightarrow \eta \quad \text { weakly in } L^{2}(0, T ; H)
\end{aligned}
$$

as $\varepsilon_{n} \rightarrow 0$ and $(u, v, \eta)$ is a solution of (1.1) satisfying the following estimates:

$$
|u|_{Y}^{2}+|v|_{Y}^{2}+|\eta|_{L^{2}\left(Q_{T}\right)}^{2} \leq C,
$$

where $C>0$ is independent of $\varepsilon, n$.

Proof Rewrite (2.3) as follows:

$$
\left\{\begin{array}{l}
u_{\varepsilon, t}-\gamma \Delta u_{\varepsilon}+\beta^{\varepsilon}\left(u_{\varepsilon}\right)+\varphi\left(u_{\varepsilon}\right)-\lambda^{\prime}\left(u_{\varepsilon}\right) v_{\varepsilon}=0 \quad \text { in } Q=\Omega \times(0, T) \\
v_{\varepsilon, t}+\lambda^{\prime}\left(u_{\varepsilon}\right) u_{\varepsilon, t}-k \Delta v_{\varepsilon}=B w_{\varepsilon} \quad \text { in } Q=\Omega \times(0, T), \\
u_{\varepsilon}(x, 0)=u_{0}(x), \quad v_{\varepsilon}(x, 0)=v_{0}(x) \quad \text { in } \Omega \\
\frac{\partial u_{\varepsilon}}{\partial v}=\frac{\partial v_{\varepsilon}}{\partial v}=0 \quad \text { on } \Sigma=\partial \Omega \times(0, T) .
\end{array}\right.
$$

Employing almost exactly the same arguments as in the proof of Lemma 2.1, we conclude the results (2.20)-(2.22). Furthermore, by a standard argument in [22], we get $\eta \in \partial I_{[-1,1]}(u)$ a.e. in $L^{2}(0, T ; H)$. This completes the proof.

Now, we assume that $\left(u^{*}, v^{*}, w^{*}\right)$ is optimal for problem $(P)$. For each $\varepsilon>0$, let $\left(u_{\varepsilon}^{*}, v_{\varepsilon}^{*}, w_{\varepsilon}^{*}\right)$ be the solution to

$$
\left\{\begin{array}{l}
u_{t}-\gamma \Delta u+\beta^{\varepsilon}(u)+\varphi(u)-\lambda^{\prime}(u) v=0 \quad \text { in } Q=\Omega \times(0, T), \\
v_{t}+\lambda^{\prime}(u) u_{t}-k \Delta v=B w^{*} \quad \text { in } Q=\Omega \times(0, T), \\
u(x, 0)=u_{0}^{*}(x), \quad v(x, 0)=v_{0}^{*}(x) \quad \text { in } \Omega, \\
\frac{\partial u}{\partial v}=\frac{\partial v}{\partial v}=0 \quad \text { on } \Sigma=\partial \Omega \times(0, T) .
\end{array}\right.
$$

It follows from Lemma 2.2 that

$$
\delta(\varepsilon) \equiv\left|u_{\varepsilon}^{*}-u^{*}\right|_{L^{2}(0, T ; H)} \rightarrow 0 .
$$

Now, the approximating optimal control problems $\left(P^{\varepsilon}\right)$ are as follows:

Minimize $L_{\varepsilon}(w) \quad$ over $w \in L^{2}(0, T ; U)$, 
where $L_{\varepsilon}: L^{2}(0, T ; U) \rightarrow R$, by

$$
L_{\varepsilon}(w)=\int_{0}^{T}\left[g_{\varepsilon}\left(t, u_{\varepsilon}\right)+h(w)\right] d t+\frac{1}{2}\left|w-w^{*}\right|_{L^{2}(0, T ; U)}^{2}+\frac{1}{2 \delta(\varepsilon)}\left[d_{S}\left(F\left(u_{\varepsilon}\right)\right)+\delta(\varepsilon)\right]^{2}
$$

and $(u, v)$ is the solution of (2.3). Here, $d_{S}(F(u))$ denotes the distance of $F(u)$ to $S$,

$$
g_{\varepsilon}(t, y)=\int_{R^{n}} g\left(t, P_{n} y-\varepsilon \Lambda_{n} \tau\right) \rho_{n}(\tau) d \tau
$$

is the approximations of $g$ [23], where $n=\left[\frac{1}{\varepsilon}\right], \rho_{n}$ is a mollifier in $R^{n}, P_{n}: H \rightarrow X_{n}$ is the projection of $H$ on $X_{n}$, which is the finite dimensional space generated by $\left\{e_{i}\right\}_{i=1}^{n},\left\{e_{i}\right\}_{i=1}^{\infty}$ is an orthonormal basis in $H, \Lambda_{n}: R^{n} \rightarrow X_{n}$ is the operator defined by $\Lambda_{n}(\tau)=\sum_{i=1}^{n} \tau_{i} e_{i}$, $\tau=\left(\tau_{1}, \tau_{2}, \ldots, \tau_{n}\right)$.

First of all, we show the existence of optimal solutions for $\left(P^{\varepsilon}\right)$.

Lemma $2.3\left(P^{\varepsilon}\right)$ has at least one optimal solution.

Proof Let $\varepsilon>0$ be fixed. It is clear that inf $L_{\varepsilon}(w)>-\infty$. Let $d_{\varepsilon}=\inf \left\{L_{\varepsilon}(w): w \in L^{2}(0, T ; U)\right\}$ and $w_{n}$ be a minimizing sequence such that

$$
d_{\varepsilon} \leq L_{\varepsilon}\left(w_{n}\right) \leq d_{\varepsilon}+\frac{1}{n}
$$

which together with $\left(\mathrm{H}_{2}\right)$, $\left(\mathrm{H}_{3}\right)$ and $(2.28)$ implies that $w_{n}$ is bounded in $L^{2}(0, T ; U)$. Without loss of generality, we may assume that $w_{n} \rightarrow \tilde{w}$ in $L^{2}(0, T ; U)$. Let $\left(u_{n}, v_{n}\right)$ and $(\tilde{u}, \tilde{v})$ be the solutions of (2.3) corresponding to $w_{n}$ and $\tilde{w}$, respectively. It follows from Lemma 2.1 that on some subsequence of $\left(u_{n}, v_{n}\right)$, still denoted by itself,

$$
\begin{aligned}
\left(u_{n}, v_{n}\right) \rightarrow(\tilde{u}, \tilde{v}) \quad & \text { weakly in } Y \times Y \text { and } \\
& \text { strongly in }\left(C\left(0, T ; H^{1}(\Omega)\right) \cap L^{2}(0, T ; H)\right)^{2} .
\end{aligned}
$$

With the help of $\left(\mathrm{H}_{2}\right),(2.29)$ and (2.31), we also obtain

$$
\int_{0}^{T}\left|g_{\varepsilon}\left(t, u_{n}\right)-g_{\varepsilon}(t, \tilde{u})\right|_{L^{2}} d t \leq C \int_{0}^{T}\left|u_{n}-\tilde{u}\right|_{L^{2}} d t \rightarrow 0 \quad \text { as } n \rightarrow \infty .
$$

On the other hand, due to $(2.31)$ and $\left(\mathrm{H}_{1}\right)$, we have that

$$
\lim _{n \rightarrow \infty} F\left(u_{n}\right)=F(\tilde{u})
$$

and therefore

$$
\lim _{n \rightarrow \infty} \frac{1}{2 \delta(\varepsilon)}\left[d_{S}\left(F\left(u_{n}\right)\right)+\delta(\varepsilon)\right]^{2}=\frac{1}{2 \delta(\varepsilon)}\left[d_{S}(F(\tilde{u}))+\delta(\varepsilon)\right]^{2} .
$$

Finally, (2.28) and (2.32)-(2.34) imply that $(\tilde{u}, \tilde{v}, \tilde{w})$ is the optimal pair for problem $\left(P^{\varepsilon}\right)$. This concludes the proof of Lemma 2.3 . 
Lemma 2.4 Let $w_{\varepsilon}$ be optimal for problem $\left(P^{\varepsilon}\right)$ and $\left(u_{\varepsilon}, v_{\varepsilon}\right)$ be the solution of $(2.3)$ corresponding to $w_{\varepsilon}$. Then, on some subsequence $\varepsilon_{n}$,

$$
\begin{aligned}
& \left(u_{\varepsilon_{n}}, v_{\varepsilon_{n}}\right) \rightarrow\left(u^{*}, v^{*}\right) \quad \text { strongly in }\left(L^{2}(0, T ; V) \cap C(0, T ; H)\right)^{2}, \\
& w_{\varepsilon_{n}} \rightarrow w^{*} \quad \text { strongly in } L^{2}(0, T ; U) .
\end{aligned}
$$

Proof Since $w_{\varepsilon}$ is a solution to $\left(P_{\varepsilon}\right)$, we have

$$
L_{\varepsilon}\left(w_{\varepsilon}\right) \leq \int_{0}^{T}\left[g_{\varepsilon}\left(t, u_{\varepsilon}^{*}\right)+h\left(w^{*}\right)\right] d t+\frac{1}{2 \delta(\varepsilon)}\left[d_{S}\left(F\left(u_{\varepsilon}^{*}\right)\right)+\delta(\varepsilon)\right]^{2},
$$

which together with (2.27) implies that

$$
\begin{aligned}
\frac{1}{2 \delta(\varepsilon)}\left[d_{S}\left(F\left(u_{\varepsilon}^{*}\right)\right)+\delta(\varepsilon)\right]^{2} & \leq \frac{1}{2 \delta(\varepsilon)}\left[\left|F\left(u_{\varepsilon}^{*}\right)-F\left(u^{*}\right)\right|_{Z}+\delta(\varepsilon)\right]^{2} \\
& \leq \frac{1}{2 \delta(\varepsilon)}\left[C\left|u_{\varepsilon}^{*}-u^{*}\right|_{L^{2}(0, T ; H)}+\delta(\varepsilon)\right]^{2} \\
& \leq \frac{(1+C)^{2}}{2} \delta(\varepsilon) \rightarrow 0 \quad \text { as } \varepsilon \rightarrow 0,
\end{aligned}
$$

which combined with (2.37) implies that

$$
\limsup _{\varepsilon \rightarrow 0} L\left(w_{\varepsilon}\right) \leq \int_{0}^{T}\left[g\left(t, u^{*}\right)+h\left(w^{*}\right)\right] d t,
$$

which implies that (2.39), that $w_{\varepsilon}$ is bounded in $L^{2}(0, T ; U)$. Without loss generality, we may assume that $w_{\varepsilon} \rightarrow \tilde{w}$ weakly in $L^{2}(0, T ; U)$, which together with Lemma 2.2 implies that there exists a sequence of $\varepsilon_{n}$ such that

$$
\left(u_{\varepsilon_{n}}, v_{\varepsilon_{n}}\right) \rightarrow(\tilde{u}, \tilde{v}) \quad \text { strongly in }\left(L^{2}(0, T ; V) \cap C(0, T ; H)\right)^{2} .
$$

On the other hand, (2.28) and (2.39) imply that

$$
\lim _{\varepsilon_{n} \rightarrow 0} d_{S}\left(F\left(u_{\varepsilon_{n}}\right)\right)=0,
$$

and therefore

$$
\lim _{\varepsilon_{n} \rightarrow 0} d_{S}(F(\tilde{u}))=0 .
$$

Thus, we conclude from (2.28), (2.40) and (2.42) that

$$
\liminf _{\varepsilon_{n} \rightarrow 0} L_{\varepsilon_{n}}\left(w_{\varepsilon_{n}}\right) \geq \int_{0}^{T}[g(t, \tilde{u})+h(\tilde{w})] d t .
$$

Finally, it follows from (2.39), (2.43) and Lemma 2.2 that

$$
\begin{aligned}
& \left(u_{\varepsilon_{n}}, v_{\varepsilon_{n}}, w_{\varepsilon_{n}}\right) \rightarrow\left(u^{*}, v^{*}, w^{*}\right) \\
& \quad \text { strongly in }\left(L^{2}(0, T ; V) \cap C(0, T ; H)\right)^{2} \times L^{2}(0, T ; U) .
\end{aligned}
$$

This completes the proof. 


\section{The optimality condition for $\left(P^{\varepsilon}\right)$ and $(P)$}

In the following we derive the optimality condition for problem $(P)$ by showing the relation between approximation problem $\left(P^{\varepsilon}\right)$ and problem $(P)$. We start this section with the necessary conditions for $w_{\varepsilon}$ to be optimal for $\left(P^{\varepsilon}\right)$.

Lemma 3.1 Suppose that $\left(\mathrm{H}_{1}\right),\left(\mathrm{H}_{2}\right),\left(\mathrm{H}_{3}\right)$ and $\left(\mathrm{H}_{4}\right)$ hold. Let $w_{\varepsilon}$ be optimal for problem $\left(P^{\varepsilon}\right)$ and $\left(u_{\varepsilon}, v_{\varepsilon}\right)$ be the solution of (2.3) corresponding to $w_{\varepsilon}$. Then there exists a tetrad $\left(\mu_{\varepsilon}, p_{\varepsilon}, q_{\varepsilon}, \zeta_{\varepsilon}\right) \in R \times L^{2}(0, T ; V) \cap C(0, T ; H) \times L^{2}(0, T ; V) \cap C(0, T ; H) \times Z^{*}$ such that

$$
\left\{\begin{array}{l}
-p_{\varepsilon, t}-\gamma \Delta p_{\varepsilon}+\beta^{\prime}\left(u_{\varepsilon}\right) p_{\varepsilon}+\left(3 u_{\varepsilon}^{2}-1\right) p_{\varepsilon}-\lambda^{\prime \prime}\left(u_{\varepsilon}\right) p_{\varepsilon} v_{\varepsilon}-\lambda^{\prime}\left(u_{\varepsilon}\right) q_{\varepsilon} \\
\quad=-\left[\partial F\left(u^{*}\right)\right]^{*} \zeta_{0}-\mu_{0} \partial g\left(t, u^{*}\right) \\
-q_{\varepsilon, t}+\lambda^{\prime}\left(u_{\varepsilon}\right) p_{\varepsilon, t}-k \Delta q_{\varepsilon}+\lambda^{\prime \prime}\left(u_{\varepsilon}\right) p_{\varepsilon} u_{\varepsilon, t}=0 \\
p(T)=0, \quad q(T)=0
\end{array}\right.
$$

$$
B^{*} q_{\varepsilon}=\mu_{\varepsilon}\left[\nabla h\left(w_{\varepsilon}\right)+w_{\varepsilon}-w^{*}\right] \text { a.e. } t \in[0, T]
$$

and

$$
\zeta_{\varepsilon} \in \partial d_{S}\left(F\left(u_{\varepsilon}\right)\right)
$$

where $\partial d_{S}$ is the sub-differential of $d_{S}$.

Proof Let $w \in L^{2}(0, T ; U), w_{\varepsilon}^{\chi}=w_{\varepsilon}+\chi w$ and $\left(u_{\varepsilon}^{\chi}, v_{\varepsilon}^{\chi}\right)$ be the solution of $(2.3)$ corresponding to $w_{\varepsilon}^{\chi}$. Then it is clear that

$$
\left(u_{\varepsilon}^{\chi}, v_{\varepsilon}^{\chi}\right) \rightarrow\left(u_{\varepsilon}, v_{\varepsilon}\right) \quad \text { strongly in } C(0, T ; H) \cap L^{2}(0, T ; V) \text { as } \chi \rightarrow 0 .
$$

Now, owing to the fact that $w_{\varepsilon}$ is optimal for problem $\left(P_{\varepsilon}\right)$, we have $\frac{L_{\varepsilon}\left(w_{\varepsilon}^{\chi}\right)-L_{\varepsilon}\left(w_{\varepsilon}\right)}{\chi} \geq 0$ (for all $\chi>0$ ), hence

$$
0 \leq \mu_{\varepsilon} \int_{0}^{T}\left\langle\nabla g_{\varepsilon}\left(t, u_{\varepsilon}\right), y_{\varepsilon}\right\rangle+\left\langle\nabla h\left(w_{\varepsilon}\right)+w_{\varepsilon}-w^{*}, w\right\rangle_{U} d t+\left\langle\left(F^{\prime}\left(u_{\varepsilon}\right)\right)^{*} \zeta_{\varepsilon}, y_{\varepsilon}\right\rangle,
$$

where $\left(y_{\varepsilon}, \bar{y}_{\varepsilon}\right)$ is the solution to

$$
\left\{\begin{array}{l}
y_{\varepsilon, t}-\gamma \Delta y_{\varepsilon}+\beta^{\prime}\left(u_{\varepsilon}\right) y_{\varepsilon}+\left(3 u_{\varepsilon}^{2}-1\right) y_{\varepsilon}-\lambda^{\prime \prime}\left(u_{\varepsilon}\right) y_{\varepsilon} v_{\varepsilon}-\lambda^{\prime}\left(u_{\varepsilon}\right) \bar{y}_{\varepsilon}=0 \\
\bar{y}_{\varepsilon, t}+\lambda^{\prime}\left(u_{\varepsilon}\right) y_{\varepsilon, t}-k \Delta \bar{y}_{\varepsilon}+\lambda^{\prime \prime}\left(u_{\varepsilon}\right) y_{\varepsilon} u_{\varepsilon, t}=0 \\
y_{\varepsilon}(0)=0, \quad \bar{y}_{\varepsilon}(0)=0
\end{array}\right.
$$

Next, employing the same arguments as in the proof of [23], we conclude that

$$
\begin{aligned}
& \lim _{\chi \rightarrow 0} \frac{1}{\chi} \int_{0}^{T} g_{\varepsilon}\left(t, u_{\varepsilon}^{\chi}\right)-g_{\varepsilon}\left(t, u_{\varepsilon}\right) d t=\int_{0}^{T}\left\langle\nabla g_{\varepsilon}\left(t, u_{\varepsilon}\right), y\right\rangle d t \\
& \lim _{\chi \rightarrow 0} \frac{1}{\chi} \int_{0}^{T}\left[\left(h\left(w_{\varepsilon}^{\chi}\right)-h\left(w_{\varepsilon}\right)\right)+\frac{1}{2}\left(\left|w_{\varepsilon}^{\chi}-w^{*}\right|_{U}^{2}-\left|w_{\varepsilon}-w^{*}\right|_{U}^{2}\right)\right] d t \\
& \quad=\int_{0}^{T}\left\langle\nabla h\left(w_{\varepsilon}\right)+w_{\varepsilon}-w^{*}, w\right\rangle d t
\end{aligned}
$$


and

$$
\lim _{\chi \rightarrow 0}\left(\left[d_{S} F\left(u_{\varepsilon}^{\chi}\right)+\varepsilon\right]^{2}-\left[d_{S} F\left(u_{\varepsilon}\right)+\varepsilon\right]^{2}\right)=\frac{F\left(u_{\varepsilon}\right)+\varepsilon}{\varepsilon}\left\langle\zeta_{\varepsilon},\left.F^{\prime}\left(u_{\varepsilon}\right) y\right|_{Z^{*}, Z^{\prime}}\right.
$$

where $\nabla g_{\varepsilon}\left(t, u_{\varepsilon}\right)$ denotes the gradient of $g_{\varepsilon}$ to the second variable at $u_{\varepsilon}$ and $\nabla h\left(w_{\varepsilon}\right)$ denotes the gradient of $h$ at $w_{\varepsilon}$. Here, $\zeta_{\varepsilon} \in \partial d_{S}\left(F\left(u_{\varepsilon}\right)\right)$ and $\partial d_{S}$ is the sub-differential of $d_{S}$, which implies (3.3). Thanks to $S$ being convex, closed and $Z^{*}$ being strictly convex, we may also infer that

$$
\left|\zeta_{\varepsilon}\right|_{Z^{*}}= \begin{cases}1 & \text { if } F\left(u_{\varepsilon}\right) \notin S \\ 0 & \text { if } F\left(u_{\varepsilon}\right) \in S\end{cases}
$$

Let

$$
\mu_{\varepsilon}=\frac{\delta(\varepsilon)}{\delta(\varepsilon)+d_{S}\left(F\left(u_{\varepsilon}\right)\right)}
$$

and $\left(p_{\varepsilon}, q_{\varepsilon}\right)$ be the solution of (3.1). It follows from (3.1), (3.5) and (3.6) that

$$
0 \leq \int_{0}^{T}-\left\langle B^{*} q_{\varepsilon}, w\right\rangle+\mu_{\varepsilon}\left\langle\nabla h\left(w_{\varepsilon}\right)+w_{\varepsilon}-w^{*}, w\right\rangle_{U} d t
$$

which implies (3.2). This completes the proof.

The proof of Theorem 1.1 By using the properties of $\alpha^{\varepsilon}$ and $\beta^{\varepsilon}$ and Lemma 2.2, we have that, on a sequence of $\varepsilon$ still denoted by $\varepsilon$,

$$
\begin{aligned}
& \left(u_{\varepsilon}, v_{\varepsilon}\right) \rightarrow(u, v) \quad \text { weakly in }\left(L^{\infty}(0, T ; V) \cap L^{2}\left(0, T ; H^{2}\right)\right)^{2}, \\
& \left(u_{\varepsilon}, v_{\varepsilon}\right) \rightarrow(u, v) \quad \text { strongly in }\left(C(0, T ; H) \cap L^{2}(0, T ; V)\right)^{2}, \\
& \left(u_{\varepsilon}^{\prime}, v_{\varepsilon}^{\prime}\right) \rightarrow\left(u^{\prime}, v^{\prime}\right) \quad \text { weakly in }\left(L^{2}(0, T ; H)\right)^{2}, \\
& \beta^{\prime}\left(u_{\varepsilon}\right) y_{\varepsilon} \rightarrow \eta \quad \text { weakly star in }\left(L^{\infty}\left(Q_{T}\right)\right)^{*} .
\end{aligned}
$$

It follows from (3.10) and (3.11) that

$$
1 \leq \mu_{\varepsilon}+\left|\zeta_{\varepsilon}\right|_{Z^{*}} \leq 2 \text { for any } \varepsilon>0
$$

Therefore, there exist generalized subsequences of $\mu_{\varepsilon}$ and $\zeta_{\varepsilon}$ such that

$$
\mu_{\varepsilon} \rightarrow \mu_{0} \quad \text { as } \varepsilon \rightarrow 0
$$

and

$$
\zeta_{\varepsilon} \rightarrow \zeta_{0} \quad \text { weakly star in } Z^{*} \text { as } \varepsilon \rightarrow 0 \text {. }
$$

Using Lemma 2.2, we may pass to the limit in (3.2) and derive (1.3) 
On the other hand, due to Lemma 2.2 and the same argument as in [23], we can conclude that

$$
\nabla g_{\varepsilon}\left(t, u_{\varepsilon}\right) \rightarrow \rho(t) \quad \text { weakly in } L^{2}(0, T ; H) \text { as } \varepsilon \rightarrow 0,
$$

where $\rho(t) \in \partial g\left(t, u^{*}\right)$ for all most $t \in(0, T)$. Thanks to $\left(\mathrm{H}_{1}\right)$ and Lemma 2.2, we also infer

$$
\left[F^{\prime}\left(u_{\varepsilon}\right)\right]^{*} \zeta_{\varepsilon} \rightarrow\left[F^{\prime}\left(u^{*}\right)\right]^{*} \zeta_{0} \quad \text { weakly in } L^{2}(0, T ; H) \text { as } \varepsilon \rightarrow 0
$$

Now we claim that

$$
\left(3 u_{\varepsilon}^{2}-1\right) p_{\varepsilon} \rightarrow\left(3\left(u^{*}\right)^{2}-1\right) p \quad \text { weakly star in } L^{2}\left(0, T ; V^{*}\right) \text { as } \varepsilon \rightarrow 0 .
$$

Indeed, let $w \in L^{2}(0, T ; V)$ and $v=\max \left\{|p|_{L^{2}(0, T ; V)}+1,\left|u^{*}\right|_{L^{2}(0, T ; V)}+1,|w|_{L^{2}(0, T ; V)}\right\}$, then we derive

$$
\begin{aligned}
& \int_{0}^{T}\left|\left(3 u_{\varepsilon}^{2}-1\right) p_{\varepsilon}-\left(3\left(u^{*}\right)^{2}-1\right) p, w\right\rangle \mid d t \\
& \quad \leq \int_{0}^{T}\left|\left\langle 3\left(u_{\varepsilon}^{2}-\left(u^{*}\right)^{2}\right) p+\left[3\left(u_{\varepsilon}\right)^{2}-1\right]\left(p_{\varepsilon}-p\right), w\right\rangle\right| d t \\
& \quad \leq \int_{0}^{T}\left|\left\langle 3\left(u_{\varepsilon}+u^{*}\right)\left(u_{\varepsilon}-u^{*}\right) p_{\varepsilon}+\left[3\left(u_{\varepsilon}\right)^{2}-1\right]\left(p_{\varepsilon}-p\right), w\right\rangle\right| d t \\
& \quad \leq 2\left(v^{6}+1\right)\left[\max _{0 \leq s \leq T}\left|u_{\varepsilon}-u^{*}\right|_{2}^{2}+\int_{0}^{T}\left|p_{\varepsilon}-p\right|_{2}^{2} d t\right] \rightarrow 0 \quad \text { as } \varepsilon \rightarrow 0 .
\end{aligned}
$$

With the help of (3.13), (3.16), (3.18)-(3.23), we can pass to the limit in (3.1) to derive that $(p, q) \in\left(L^{2}(0, T ; V) \cap C(0, T ; H)\right)^{2}$ and satisfies (1.2). On the other hand, observing that $\zeta_{\varepsilon} \in \partial d_{S}\left(F\left(u_{\varepsilon}\right)\right)$, we derive

$$
\left\langle\zeta_{\varepsilon}, w-\left.F\left(u^{*}\right)\right|_{Z^{*}, Z} \leq\left\langle\zeta_{\varepsilon}, F\left(u^{*}\right)-\left.F\left(u_{\varepsilon}\right)\right|_{Z^{*}, Z}\right.\right.
$$

which together with (3.19) and Lemma 2.2 implies (1.3) $)_{2}$ (the second inequality of (1.3)).

Finally, we are in a position to prove that $\left(\mu_{0}, \zeta_{0}\right) \neq 0$. To this end, we suppose that $\mu_{0}=0$. It follows from (3.17), (3.19) and (3.24) that there exist $\varepsilon_{1}>0$ and $\delta>0$ such that

$$
\delta \leq\left|\zeta_{\varepsilon}\right|_{Z^{*}} \leq 2 \text { for any } \varepsilon<\varepsilon_{1}
$$

and

$$
\left\langle\zeta_{\varepsilon}, w-F\left(u^{*}\right)\right\rangle_{Z^{*}, Z} \leq\left\langle\zeta_{\varepsilon}, F\left(u^{*}\right)-\left.F\left(u_{\varepsilon}\right)\right|_{Z^{*}, Z} \rightarrow 0 \quad \text { uniformly in } w \in S\right. \text {. }
$$

Since $S \subset X$ is a closed convex subset with finite co-dimensionality, so is $S-F\left(u^{*}\right)$, which together with (3.25) and (3.26) implies that $\left(\mu_{0}, \zeta_{0}\right) \neq 0$ [24].

Assuming that $F^{\prime}\left(u^{*}\right)$ is injective and $\left(\mu_{0}, p, q\right)=0$, thanks to (1.2), we derive $\left(F^{\prime}\left(u^{*}\right)\right)^{*} \zeta_{0}=$ 0 , which yields $\zeta_{0}=0$ and $\left(\mu_{0}, \zeta_{0}\right)=0$. This is a contradiction with $\left(\mu_{0}, \zeta_{0}\right) \neq 0$. Thus, if $F^{\prime}\left(u^{*}\right)$ is injective, then $\left(\mu_{0}, p\right) \neq 0$. We complete the proof. 


\section{Competing interests}

The authors declare that they have no competing interests.

\section{Authors' contributions}

$J Z$ carried out the optimal control problem for the phase-field transition system with state constraint and obstacle and drafted the manuscript. HL and JL participated in the design of the study and examined the results carefully. All authors read and approved the final manuscript.

\section{Author details}

'School of Mathematics, Beijing Institute of Technology, Beijing, China. ${ }^{2}$ School of Science, University of Science and Technology Liaoning, Anshan, 114051, China.

\section{Received: 23 June 2013 Accepted: 25 September 2013 Published: 08 Nov 2013}

\section{References}

1. Temam, R: Infinite-Dimensional Dynamical Systems in Mechanics and Physics, 2nd edn. Applied Mathematical Sciences. Springer, New York (1997)

2. Elliott, C, Zheng, S: Global existence and stability of solutions to the phase field equations. In: Free Boundary Problems. Intern. Ser. Numer. Math., vol. 95, pp. 48-58. Birkhäuser, Basel (1990)

3. Penrose, O, Fife, PC: Thermodynamically consistent models of phase-field type for the kinetics of phase transitions. Physica D 43, 44-62 (1990)

4. Damlamian, A, Kenmochi, N, Sato, N: Phase field equations with constraints. In: Nonlinear Mathematical Problems in Industry. Gakuto. Inter. Ser. Math. Sci. Appl., pp. 391-404. Gakkotosho, Tokyo (1993)

5. Damlamian, AL, Kenmochi, N: Evolution equations associated with non-isothermal phase separation: subdifferential approach. Ann. Mat. Pura Appl. 4, 167-190 (1999)

6. Ito, A, Kenmochi, N: Asymptotic behaviour of solutions to phase field models with constraints. Funkc. Ekvacioj 39 123-142 (1996)

7. Ito, A, Kenmochi, N: Asymptotic behaviour of solutions to non-isothermal phase separation model with constraint in one-dimensional space. J. Math. Soc. Jpn. 50,491-519 (1998)

8. Kenmochi, N: Systems of nonlinear PDEs arising from dynamical phase transitions. In Visintin, A (ed.) Phase Transitions and Hysteresis. Lecture Notes in Math., vol. 1584, pp. 39-86. Springer, Berlin (1994)

9. Conti, M, Gatti, S, Miranville, A: Asymptotic behavior of the Caginalp phase-field system with coupled dynamic boundary conditions. Discrete Contin. Dyn. Syst., Ser. S 5, 485-505 (2012)

10. Grasselli, M, Miranville, A, Schimperna, G: The Caginalp phase-field system with coupled dynamic boundary conditions and singular potentials. Discrete Contin. Dyn. Syst. 28, 67-98 (2010)

11. Barbu, V: Local controllability of the phase field system. Nonlinear Anal., Theory Methods Appl. 50, 363-372 (2002)

12. Colli, P, Gilardi, G, Podio-Guidugli, P, Sprekels, J: Distributed optimal control of a nonstandard system of phase field equations. Contin. Mech. Thermodyn. 24, 437-459 (2012)

13. Chen, Z: Optimal boundary controls for a phase field model. IMA J. Math. Control Inf. 10, 157-176 (1993)

14. Lefter, C, Sprekels, J: Optimal boundary control of a phase field system modeling non-isothermal phase transitions. Adv. Math. Sci. Appl. 17, 181-194 (2007)

15. Moroşanu, C, Wang, G: State-constrained optimal control for the phase-field transition system. Numer. Funct. Anal. Optim. 28, 379-403 (2007)

16. Ryu, S-U, Yagi, A: Optimal control for an adsorbate-induced phase transition model. Appl. Math. Comput. 171 420-432 (2005)

17. Benincasa, T, Favini, A, Moroşanu, C: A product formula approach to a nonhomogeneous boundary optimal control problem governed by nonlinear phase-field transition system part II: Lie-Trotter product formula. J. Optim. Theory Appl. 148, 31-45 (2011)

18. Horn, W, Sokolowskiy, J, Sprekels, J: Control problems with state constraints for the Penrose-Fife phase-field model Weierstrass-Inst. für Angewandte Analysis und Stochastik (1995)

19. Wang, Q, Nakagiri, S: Research Institute for Mathematical Sciences Kokyuüroku 1128, 172-180 (2000)

20. Zheng, S: Global existence for a thermodynamically consistent model of phase field type. Differ. Integral Equ. 5, 241-253 (1992)

21. Ohtsuka, T, Shirakawa, K, Yamazaki, N: Optimal control problem for Allen-Cahn type equation associated with total variation energy. Discrete Contin. Dyn. Syst. 5, 159-181 (2012)

22. Barbu, V: Analysis and control of nonlinear infinite dimensional systems. In: Ames, WF (ed.) Mathematics in Science and Engineering. Georgia Institute of Technology (1993)

23. Barbu, V: Optimal Control of Variational Inequalities. Pitman Research Notes in Mathematics, Boston (1984)

24. Lions, JL: Optimal Control of Systems Governed by Partial Differential Equations. Springer, Berlin (1971)

10.1186/1687-2770-2013-234

Cite this article as: Zheng et al.: State-constrained optimal control of phase-field equations with obstacle. Boundary Value Problems 2013, 2013:234 